\title{
Performance in a novel environment subject to ghost competition
}

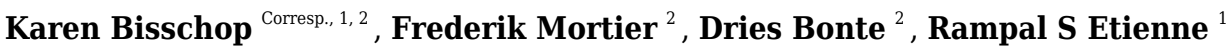 \\ ${ }^{1}$ Groningen Institute for Evolutionary Life Sciences, University of Groningen, Groningen, Netherlands \\ 2 Department of Biology, Universiteit Gent, Ghent, Belgium \\ Corresponding Author: Karen Bisschop \\ Email address: kbisschop.evo@gmail.com
}

Background. A central tenet of the evolutionary theory of communities is that competition impacts evolutionary processes such as local adaptation. Species in a community exert a selection pressure on other species and may drive them to extinction. We know, however, very little about the influence of unsuccessful or ghost species on the evolutionary dynamics within the community.

Methods. Here we report the long-term influence of a ghost competitor on the performance of a more successful species using experimental evolution. We transferred the spider mite Tetranychus urticae onto a novel host plant under initial presence or absence of a competing species, the congeneric mite $T$. ludeni.

Results. The competitor species, T. ludeni, unintentionally went extinct soon after the start of the experiment, but we nevertheless completed the experiment and found that the early competitive pressure of this ghost competitor positively affected the performance (i.e. fecundity) of the surviving species, $T$. urticae. This effect on $T$. urticae lasted for at least 25 generations.

Discussion. Our study suggests that early experienced selection pressures can exert a persistent evolutionary signal on species' performance in novel environments. 
1 Performance in a novel environment subject to ghost competition.

2 Karen Bisschop ${ }^{1,2}$, Frederik Mortier ${ }^{2}$, Dries Bonte ${ }^{2} \dot{\sharp}$, Rampal S. Etienne $^{1 \ddagger}$

$3 \mp \quad$ Joint last authorship

41 Groningen Institute for Evolutionary Life Sciences, University of Groningen, Groningen,

5 The Netherlands

62 Department of Biology, Universiteit Gent, Ghent, Belgium

7

8 Corresponding author:

9 Karen Bisschop ${ }^{1,2}$

10

11 Email address: kbisschop.evo@gmail.com 


\section{ABSTRACT}

13 Background. A central tenet of the evolutionary theory of communities is that competition impacts evolutionary processes such as local adaptation. Species in a community exert a

15 selection pressure on other species and may drive them to extinction. We know, however, very

16 little about the influence of unsuccessful or ghost species on the evolutionary dynamics within

17 the community.

18 Methods. Here we report the long-term influence of a ghost competitor on the performance of a more successful species using experimental evolution. We transferred the spider mite Tetranychus urticae onto a novel host plant under initial presence or absence of a competing

21 species, the congeneric mite T. ludeni.

22 Results. The competitor species, T. ludeni, unintentionally went extinct soon after the start of the experiment, but we nevertheless completed the experiment and found that the early competitive pressure of this ghost competitor positively affected the performance (i.e. fecundity) of the

25 surviving species, T. urticae. This effect on T. urticae lasted for at least 25 generations.

Discussion. Our study suggests that early experienced selection pressures can exert a persistent

27 evolutionary signal on species' performance in novel environments. 
29 Populations are facing a continuously changing world that they can possibly cope with in various

30

31

32

ways, such as through phenotypic plasticity or by tracking their favoured habitat. If these

solutions are not possible, evolutionary rescue by genetic adaptation may eventually allow persistence (Lindsey et al., 2013). One factor influencing this local adaptation is competition. Interspecific competition is known to influence local adaptation in many different ways, but the effect is still largely unpredictable (Rice \& Knapp, 2008; Alzate et al., 2017; Zhao et al., 2018). First, heterospecific competitors might modify the selection pressure exerted by the abiotic environment, enhancing or limiting genetic adaptation to the novel environment (Osmond \& de Mazancourt, 2013). Classical examples of enhanced genetic adaptation are seen in adaptive radiations of three-spined sticklebacks or fast character displacements in Darwin finches or Myzomelid honeyeaters (Diamond et al., 1989; Schluter, 1994; Reznick \& Ghalambor, 2001). Previously, we found that additional selection pressure exerted by a congeneric species ( $T$. evansi) facilitated adaptation of the focal species (T. urticae) to a novel environment under high dispersal from a maladapted ancestral population (Alzate et al., 2017). Adaptation to the novel environment can also be reduced by interspecific competition when there is, for instance, a tradeoff between traits responsible for adaptation to the competing species and to the novel environment (Chesson, 2000; Siepielski et al., 2016).

Furthermore, interspecific competition can create new niches or change the current environment for species to adapt to. Species may use waste products or adapt to plants with modified defences caused by co-occurring individuals (Sarmento et al., 2011; Lawrence et al., 2012). These new niches will subsequently create opportunities for adaptive shifts to novel environmental 
50 conditions. This illustrates that competition and facilitation can jointly shape evolution, making it

51 difficult to study the consequences of interspecific competition alone.

52 As a last scenario, interspecific competition can hinder or limit the process of local adaptation by

53 restricting resource availability and hence decrease effective population size. The resulting

54 increased probabilities of genetic drift will then decrease the evolutionary potential and hence the

55 chance of local adaptation (Lawrence et al., 2012; Osmond \& de Mazancourt, 2013; Zhao et al.,

56 2018). Extreme hindrance to local adaptation can even cause extinction of one of the species

57 (Jaeger, 1970; Bengtsson, 1989; Griffis \& Jaeger, 1998).

58

In this study we aimed to further unravel the effect of interspecific competition on adaptation to a novel food source. We therefore performed an evolutionary experiment with two related spider mite species, Tetranychus urticae and T. ludeni, adapting to a novel host. Both species were placed alone or together on a new host plant and we wanted to study how this interspecific competition affects local adaptation. More precisely, we wanted to test for differences in the rates of adaptation and the final performance on the novel food source at the end of the experiment. The two competitors were supposed to be competitively similar, but the experiment demonstrated that this was not the case: both species could only temporarily co-occur, because $T$. ludeni went extinct after a few generations. We nevertheless continued the experiment and conducted subsequent analyses that provided convincing evidence for the effect of the ghost competition on adaptation. More precisely, we focused on the surviving species, T. urticae, and explored whether we could detect long-term evolutionary effects on performance (measured as fecundity) of this species due to differences in early selection pressures caused by the ghost competitor, T. ludeni. We chose the average of the initial population size of the unsuccessful 
73 species during the first month of co-occurrence as an indication for the early competitive

74 pressure. These differences in population sizes arose naturally and can be attributed to selection,

75 as well as drift and founder effects.

76 While inferior competitors are predicted to eventually go extinct, they may co-occur with the

77 more successful competitors for many generations (Holmes \& Wilson, 1998; Lankau, 2011).

78 These early and non-persisting interactions may leave a strong signature on the future

79 community dynamics (Law \& Daniel Morton, 1996; Miller, TerHorst \& Burns, 2009; Mallon et

80 al., 2018), because they have the possibility to induce large habitat modifications or evolutionary

81 changes in the more successful species. Historical contingency (i.e. the influence of the arrival

82 time of a certain species in a community; Fukami 2015) in terms of limitations imposed by so-

83 called ghost species (Hawkes \& Keitt, 2015) may thus have a strong impact on the eco-

84 evolutionary trajectories of populations and communities, in the same way as successful species

85 do (Fukami, 2015). The role of competition intensity of an inferior species prior to its extinction

86 on the ecological and evolutionary dynamics of persisting species is still largely unknown,

87 however.

88 In this study we found a lower fecundity of T. ludeni on bean and cucumber than T. urticae in the control populations, which may explain their rapid extinction. Still, the ghost species T. ludeni

90 showed an effect on the surviving species $T$. urticae, because the eventually achieved strength of

91 adaptation of $T$. urticae increased with the initial density of $T$. ludeni. We therefore suggest that

92 ghost competition may lead to differences in long-term local adaptation.

93

94

95 
97 Study species

98

We used two species of the family Tetranychidae (Acari, Arachnida): Tetranychus urticae Koch, 1836, and T. ludeni Zacher, 1913. These herbivorous mite species are highly suitable for evolutionary experiments due to their small body sizes, their possibility to maintain large populations in the lab, and short generation times (Zhang, 2003).

For this study, we used different inbred populations of T. urticae from Bitume and colleagues (2013). Each population originated from two adult females from the LS-VL line (Van Leeuwen, Stillatus \& Tirry, 2004) and was afterwards kept at low population densities. The LS-VL line was collected from roses in October 2000 (Ghent, Belgium). After this initial collection, all populations were maintained on bean plants (Phaseolus vulgaris, Prelude).

We used two populations of T. ludeni: the Tl Alval (Lisbon, Portugal) and Tl CVM (Lourinhã, Portugal). Both populations were sampled early autumn 2013 from common morning-glory and afterwards maintained on bean plants (P. vulgaris, Prelude). The founder populations were 160 and 300 individuals for Tl Alval and Tl CVM respectively. Our evolutionary experiment started in September 2015, implying that T. urticae and T. ludeni had been under laboratory conditions for approximately fifteen and two years, respectively.

For this study, we chose to subject different inbred lines of T. urticae that were created in 2012 (Bitume et al., 2013) to further inbreeding by mother-son mating for one more generation prior to the experiments. This resulted in the creation of 13 isofemale lines. The genetic variation within the lines is therefore very low, but larger among lines as we used different inbred lines from Bitume and colleagues (2013). It may sound counterintuitive to use inbred populations for 
119 an evolutionary experiment that mainly uses standing genetic variation (note that no spider mites

120 were added during our experiment), but in this way we could generate genetically similar

121 replicates and control for putative initial drift effects by differences in starting genetic variation.

122 We deemed this more important than potential inbreeding effects, because no effects of

123 inbreeding on genetic trait variation have been found in these and other lines (Van Petegem et

124 al., 2018). Furthermore, we created six isofemale lines for T. ludeni (coming from Tl Alval and

125 Tl CVM). We wanted to create 13 lines for this species as well, but were unsuccessful due to low

126 fertility or early mortality. In hindsight, this may partly explain the rapid extinction T. ludeni:

127 even the six initially surviving lines were probably far from optimal.

128 We created a control population of T. ludeni from the stock (Tl Alval and Tl CVM) and placed

129 them on bean plants (four two-weeks-old plants). We created a control population of T. urticae

130 from the created 13 isofemale lines (four mites per line) and placed them also on bean plants

131 (four two-weeks-old plants). All populations were kept in a climate-controlled room $\left(25^{\circ} \mathrm{C}-\right.$

$\left.13230^{\circ} \mathrm{C}, 16: 8 \mathrm{~L}: \mathrm{D}\right)$.

133

134 Experimental set-up

135 Novel host islands were created by placing two three-weeks-old cucumber plants, Cucumis

136 sativus Tanja, in boxes with yellow sticky paper (Pherobank) at the bottom and Vaseline at the

137 walls to avoid contamination between islands; this method is known to work from previous

138 research (Alzate et al., 2017; Alzate, Etienne \& Bonte, 2019; Bisschop et al., 2019). These units

139 consisting of multiple plants are referred to as 'islands' because they represent isolated habitat.

140 They represent continuous habitat because the leaves were overlapping allowing easy dispersal

141 across the plants. After the first week, two fresh three-weeks-old cucumber plants were added to 
142 create the island size of the experiment. To provide enough food for the spider mites, the islands

143 were then weekly refreshed by replacing the two oldest plants with two new three-weeks-old

144 cucumber plants. In this way, sufficient time was provided for a generation of spider mites to

145 develop on the new plants, while allowing the population to move toward the fresh leaves. The

146 removed old plants may have contained mites or unhatched eggs, but we nevertheless chose this

147 refreshment procedure to maintain relatively natural movement dynamics. It is for instance

148 known that especially young fertilised females disperse more (Li \& Margolies, 1993) and

149 dispersive individuals may differ in their body condition or performance compared to sedentary

150 individuals (Bonte et al., 2014; Dahirel et al., 2019). This refreshment procedure may have

151 caused an extra competitive pressure if one species was more dispersive or delayed its dispersal

152 for avoiding competition, but we preferred to design the experiment in a way that it resembled

153 more the actual life strategy of spider mites (colonisation with few founders followed by rapid

154 growth).

155

156 The spider mite isofemale lines (as subscribed under 'study species') were placed on the islands

157 with or without heterospecifics. Eight replicate islands received both T. urticae and T. ludeni.

158 Eight replicate islands received only T. urticae and another eight replicate islands received only

159 T. ludeni. Each island started with the same total population size of 52 adult females and as

160 similar as possible gene pool. Therefore, the group with only $T$. urticae received four adult

161 females from each of the thirteen isofemale lines. The group with only T. ludeni received four

162 adult females from the six isofemale lines and was supplemented with 28 females from its stock

163 population, because of the lack of success of creating more isofemale lines. The group with both

164 spider mite species received 26 adult females of $T$. urticae (two from each of the isofemale lines) 
165 and 26 adult females of $T$. ludeni (twelve $T$. ludeni females per island came from the six 166 isofemale lines and were supplemented with 14 mites from its stock population). Control

167 populations on bean were created as explained under 'study species'.

168 The use of the outbred stock population of T. ludeni to supplement the populations provided an 169 unanticipated opportunity as it increased the initial genetic variation of T. ludeni among 170 replicates and hence differences in early selection pressures on T. urticae.

171 We started with a relatively low population size to make it biologically relevant as natural

172 populations usually colonise plants at small population sizes. All adult female mites were equally 173 distributed over the plants.

174 We chose the same total population size and no differences among island sizes, as it is known 175 that differences in densities change both the intra- and interspecific competitive pressure and that 176 an increase in island size would change the adaptive potential of the treatment (Alzate, Etienne \& 177 Bonte, 2019). This necessarily meant that the initial population size for each species was not the 178 same across the different treatments; we recognise that this may affect genetic drift and cause 179 sampling effects, which we will come back to in the Discussion. A schematic overview of the 180 different treatments is provided in Fig. 1.

182 The total experiment lasted for ten months, which is approximately 25 generations and long 183 enough to detect local adaptation (Gould, 1979; Fry, 1989; Magalhães et al., 2007, 2009; Bonte 184 et al., 2010). For logistical reasons the experiment was performed in two blocks with one month 185 difference, each block consisted of four replicate islands per treatment.

$\underline{\text { Measurements }}$ 
188 Ecological dynamics (population density assessment)

189 Every two weeks, the density of the spider mites in the evolutionary experiment was measured

190 by counting adult females on a square of $1 \times 1 \mathrm{~cm}^{2}$; the first counting was done after two weeks.

191 The location of the square was chosen right next to the stalk of the highest, fully grown leaf of

192 the two newest plants of each island, keeping in mind that the mites had one week to move from

193 the old plants towards the fresh plants before counting. Both the abaxial as well as the adaxial

194 side were measured and summed for a total overview. The location on the leaf was chosen to

195 standardise the measurements in time and make them comparable. The populations of T. ludeni

196 under competition with T. urticae went extinct after approximately two months. To get an

197 impression of its competitive pressure on the more successful T. urticae populations while it was

198 still present, we used the mean population density of the first month of T. ludeni (hereafter called

199 'early competitive pressure of the ghost competitor').

200

201 Evolutionary dynamics (fecundity assessment)

202 Fecundity tests for the control populations on bean and for the experimental cucumber

203 populations were performed every two months to determine the level of adaptation. As the

204 experimental populations of $T$. ludeni went extinct under competition, we obviously only have

205 results from fecundity tests on the control population of $T$. ludeni. We chose fecundity as a proxy

206 of adaptation because previous research confirmed it to be the best predictor of adaptation

207 compared to survival or development (Magalhães et al., 2007; Alzate et al., 2017; Alzate,

208 Etienne \& Bonte, 2019). Five adult females were sampled from each island and separately placed

209 on a bean leaf disc $\left(17 \times 27 \mathrm{~mm}^{2}\right)$ for two generations of common-garden to standardise juvenile

210 and maternal effects (Magalhães et al., 2011; Kawecki et al., 2012). Bean discs were chosen 
211 because bean plants are very suitable host plants with a low selection pressure and will not cause

212 a change in allele frequencies of the evolved lines (Magalhães et al., 2011). These leaf discs were

213 placed in a petri dish on wet cotton wool and surrounded with paper strip borders. Then, the

214 fecundity of two quiescent deutonymph females that originated from the same common-garden

215 replicate was tested. One female was put on a bean leaf and one on a cucumber leaf (same set-up

216 as for common garden) in a climate cabinet of $30^{\circ} \mathrm{C}$ under 16:8 L:D. Bean and cucumber plants

217 were grown specifically for this test (for two and three weeks respectively) and were protected

218 against herbivory before the test. Fecundity (number of eggs laid after six days) was measured

219 based on daily pictures taken. Females that drowned in the cotton before the sixth day were

220 excluded from the analysis (this was $13.5 \%$ for the populations of T. urticae without T. ludeni,

$22115 \%$ for the populations of T. urticae with the ghost competitor, and $10.5 \%$ for the populations

222 of T. urticae in the control treatment maintained on bean). The cucumber plants that were

223 necessary for the leaf discs for the fecundity test after four months did not grow for one of our

224 two experimental blocks. Therefore, we were not able to test fecundity at that time point for

225 these replicates. In total, the fecundity was measured for 974 females (exact sample sizes per

226 treatments are given in the electronic supplementary material Table S1).

228 Statistical analysis

229 We used general linear mixed models (GLMMs; except for the dynamics and performance of the 230 ghost competitor where a GLM was used) with Negative Binomial distribution with log link to 231 account for overdispersion for both the fecundity and population density measures. The variance 232 was determined as $\mu *(1+\mu / k)$ in which $\mu$ is the mean and $k$ is the overdispersion parameter 
233 (standard negative binomial parametrisation) (Hardin \& Hilbe, 2007). The violin plots in the

234 figures illustrate the amount of overdispersion in the data.

235

236 The dynamics and performance of the ghost competitor

237 We first studied the performance of the control populations that had been maintained on bean of

238 both species on bean and cucumber. The dependent variable in the maximal model was fecundity

239 (number of eggs after six days) and the explanatory categorical variables were the plant species

240 during the fecundity assessment (bean or cucumber) and the mite species (T. urticae or $T$.

241 ludeni). Model selection was based on the lowest AICc and a Wald $\chi 2$ test was performed on the

242 maximal model to check the reliability of the model selection. We present below the results of

243 the best-fitting model. Pairwise comparisons for the variables in this best-fitting model were

244 adjusted for multiple comparisons with Tukey's method.

246 Signature of the ghost competitor on performance of T. urticae

247 Evolutionary dynamics (fecundity assessment)

248 We investigated the impact of the density of T. ludeni and of T. urticae at the onset of the

249 experiment (i.e., mean density during the first month) on the fecundity of T. urticae on its initial

250 and novel host plant. The explanatory variables in the maximal model were time (as categorical

251 variable; 2, 4, 6, 8 or 10 months), the density of $T$. ludeni (continuous variable), the density of $T$.

252 urticae (continuous variable), and the interaction between time and densities of both species. In

253 this way, we aimed to determine whether it was the own density or the density of the ghost

254 competitor that affected performance of $T$ urticae. We compared this with an additional model

255 with the total density (summing the density of T. ludeni and T. urticae), time, and their 
256 interaction to find out whether fecundity was affected by the species' individual densities or just

257 the total density. The random effect for all models was the replicate island nested within the

258 experimental blocks. However, for the assessment of fecundity on cucumber the nestedness in

259 the random effects led to overfitting of the full model, as the random effect variance was

260 estimated to be zero (Magnusson et al., 2018). As a consequence, we only used replicate island

261 as random variable for the assessment on cucumber. We chose a categorical variable for time

262 instead of a continuous one, because differences in quality of leaves at the different

263 measurements were likely and we did not want to assume a linear response of adaptation. For

264 completeness, we also performed the analyses with time as a continuous variable; the results are

265 similar, see Supplementary information (Table S2-S6). Model selection was based on the lowest

266 AICc and an additional Wald $\chi 2$ test was performed on the maximal model. We present the

267 results of this best-fitting model in the main text. Pairwise comparisons for the slopes and means

268 in this best-fitting model were adjusted for multiple comparisons with Tukey's method

269 (summary statistics for full models are provided in the electronic supplementary material Table

270 S7).

271

272 Ecological dynamics (population density assessment)

273 We used the density assessed through time of the different spider mite populations to investigate

274 differences in demography between the species with or without competitor after the plateau

275 phase (starting from day 200 based on visual inspection). The dependent variable in the maximal

276 model was the density (number of adult female mites per $\mathrm{cm}^{2}$ ) and the explanatory variable was

277 the treatment (T. urticae with and without competitor, and T. ludeni with and without

278 competitor). The random effects were time and the different island replicates within their 
279 experimental block. Model selection, Wald $\chi^{2}$ test, and pairwise comparisons were performed as 280 explained above.

281

282 Performance of $\mathrm{T}$. urticae

283 Because we were interested in the magnitude of the differences in performance due to the 284 presence of $T$. ludeni, we did a further analysis including also the control population of T. urticae 285 on bean and the populations of T. urticae on cucumber without T. ludeni. We investigated the 286 fecundity as a function of the three different treatments (control of T. urticae on bean, the 287 populations of T. urticae on cucumber without T. ludeni, and those with T. ludeni) and time 288 (categorical variable; 2, 4, 6, 8, or 10 months), and their interactions. The replicate islands were 289 treated as random effects and were nested within the two experimental blocks. Model selection, 290 Wald $\chi^{2}$ test of the maximal model, and pairwise comparisons of the variables for the best-fitting 291 model were performed as explained above. Only results of the best-fitting model are presented 292 below (summary statistics for full models are provided in the electronic supplementary material 293 Table S7).

The estimates provided in the tables are the raw and untransformed estimates for the fixed effects of the final models (negative binomial distribution). All analyses were performed in R (version

297 3.6.0) with glmmTMB version 0.2.3 (Brooks et al., 2017), MuMIn version 1.43.6 (Bartoń, 2019), 298 and emmeans version 1.3.5.1 (Lenth, 2019). 
299 RESULTS

300 The dynamics and performance of the ghost competitor

301 In the competition treatments $T$. ludeni went extinct after approximately two months. While $T$.

302 ludeni was able to maintain a population on cucumber in the absence of a competing species

303 (12.3 individuals $\left./ \mathrm{cm}^{2}\right)$, it reached a significantly lower density than T. urticae (19.8

304 individuals $/ \mathrm{cm}^{2}, t$ ratio $=7.299$ and $p<0.0001$ for $T$. urticae under ghost competition; 20.5

305 individuals $/ \mathrm{cm}^{2}, t$ ratio $=7.756$ and $p<0.0001$ for $T$. urticae without competitor). Although the

306 significantly lower performance of $T$. ludeni during the fecundity assessment could reflect

307 difficulties dealing with the host plant, competitor T. ludeni was able to maintain a population on

308 cucumber in the absence of T. urticae. This indicates that the presence of T. urticae hindered the

309 survival of T. ludeni (Fig. 2a, Fig. 2b, and Table 1-3).

310 The fecundity assessments on the initial and novel host plant with mites from the control

311 populations of T. urticae and T. ludeni (which had been maintained on bean plants and had never

312 been on cucumber before) showed that T. urticae had a significantly lower fecundity on

313 cucumber than on bean (27.9 versus 48.0 eggs after six days; $t$ ratio $=-3.629$ and $p=0.0025)$,

314 while there was no difference in the performance of T. ludeni on bean or cucumber (13.3 vs. 16.2

315 eggs after six days; $t$ ratio $=-1.012$ and $p=0.7426$ ). The fecundity assessments also showed that

316 T. ludeni laid significantly fewer eggs than T. urticae on both bean (13.3 versus 48.0 eggs after

317 six days; $t$ ratio $=-7.463$ and $p<0.0001)$ and cucumber $(16.2$ versus 27.9 eggs after six days; $t$

318 ratio $=-6.177$ and $p<0.0001)$. This suggests that the fecundity of $T$. ludeni on the novel host

319 was already lower than that of T. urticae at the onset of the experiment (Fig. 2c, Table 1-4).

320

321 Signature of the ghost competitor on performance of $\mathrm{T}$. urticae 
322 Throughout the evolutionary experiment, we measured the densities of the populations of both

323 spider mite species. During the first month, the ghost competitor (T. ludeni) was still present and

324 the early competitive pressure calculated during the first month gave an indication of the

325 pressure exerted by the ghost species on T. urticae. We found that the early competitive pressure

326 of the ghost competitor positively affected the evolved individual fecundity of T. urticae on the

327 novel host plant, cucumber, during the fecundity assessment on cucumber (Fig. 3b; $z$ value $=$

3282.33 and $p=0.0199)$. The evolved fecundity of individuals from populations under lower

329 competitive pressures was lower than for individuals from populations under a higher early

330 competitive pressure of T. ludeni (26.8 eggs after six days for T. urticae under a density of one

331 individual of T. ludeni per $\mathrm{cm}^{2}$ compared to 33.1 eggs with four individuals of $T$. ludeni per $\mathrm{cm}^{2}$ ).

332 This effect emerged from the start of the experiment and was independent of time as the best-

333 fitting model did not include time.

334 An extra analysis was performed to investigate whether excluding the first months from the 335 dataset still gave the same results (electronic supplementary material Table S8). The early 336 competitive pressure of the ghost competitor was still included as the only explanatory variable

337 in the best-fitting model when excluding the second month, both the second and fourth months, 338 and the second, fourth and sixth months. When we considered each time point by itself, this 339 result was not found, which is likely a lack of power.

340 The early competitive pressure of the ghost competitor did not explain the fecundity assessed on

341 the original host plant, bean (not included in the best-fitting model). Also, the density of $T$.

342 urticae itself or the total initial density was not related to performance during the fecundity

343 assessments on both bean and cucumber leaf discs (Table 1-5). 
344 The early competitive pressure of the ghost competitor did not influence the demography of $T$.

345 urticae. All T. urticae populations reached similar densities during the plateau phase regardless

346 of the initial presence of the ghost competitor $\left(19.8\right.$ and $20.5 \mathrm{ind} . / \mathrm{cm}^{2}$ respectively; $t$ ratio $=$ -

3470.666 and $p=0.7833$ ). At the start, the density of $T$. urticae without competition was temporarily

348 higher than the populations of T. urticae with T. ludeni present (Fig. 2a), which is most likely

349 due to the differences in starting densities of $T$. urticae between both treatments.

350

351 Performance of T. urticae

352 We compared the performance of mites from a control population that was maintained on bean

353 plants with mites adapting to cucumber where in both cases the interspecific competitor was

354 replaced by conspecifics. The evolved individual fecundity of the control population was

355 significantly lower for the assessment on cucumber than the evolved individual fecundity of

356 populations grown on cucumber (28.9 versus 33.9 eggs after six days; $t$ ratio $=-3.110$ and $p=$

357 0.0056), which suggests local adaptation to the novel host plant for the latter group (Fig. 3b;

358 Table 1-4). 
360 The process of genetic adaptation to novel environmental conditions is typically studied and

361 understood from the perspective of the available genetic variation and selection pressures as

362 imposed by the environment. Because competing species are an intrinsic part of novel

363 experienced environmental conditions, they are known to mediate sometimes complex

364 evolutionary processes. Here we provide empirical evidence that initial competition between two species can have a long-lasting effect on their performance in a novel environment.

The unintentional rapid extinction of $T$. ludeni seems a logical consequence of the higher attained fecundity of T. urticae on the novel host already at the onset of the experiment (Fig. 2c). This higher fecundity and hence higher growth rate increased the chance for better establishment or recovery after disturbance (Turcotte, Reznick \& Hare, 2011, 2013). Also, populations from $T$. urticae were at a higher density at the plateau phase than populations from T. ludeni (Fig. 2b).

372 The density of T. urticae on the measured surface was almost fifty percent more than the density of T. ludeni when grown alone. This suggests that T. urticae has a higher resource efficiency than T. ludeni, which could for instance arise from evolved detoxification mechanisms as often found between herbivores and their hosts (Després, David \& Gallet, 2007; Dermauw et al., 2018). The population size at the plateau phase might not only be due to a signature of ecological dynamics, but may also be a consequence of adaptation itself, because the carrying capacity may be affected by organismal traits. In this case, our results suggest selection for growth rate but not

379 for carrying capacity due to ghost competition. After one month the density of populations of $T$. urticae without heterospecific competition was higher than that of populations with heterospecific competition, but this difference vanished together with the extinction of the 
382 competitor. This probably means that the ghost competitor (when still present) decreased the

383 available resources resulting in a lower population size for T. urticae (Fig. 2a). Another

384 explanation is a delayed growth due to the lower initial population size in the experiment.

385

386 We have shown that the higher the competitive pressure of the ghost competitor (as measured by

387 its average density in the first month), the higher the fecundity of T. urticae was on the novel

388 host plant (Fig. 3b). We speculate that a higher selection pressure was exerted under a higher

389 early competitive pressure of the ghost competitor, which eventually led to an increase in

390 fecundity of the focal species. It is known that the competitor, T. ludeni, can down-regulate plant

391 defences (Godinho et al. 2016), but this cannot explain the correlation between its density and

392 the fecundity of the focal species even long after the ghost competitor went extinct, because

393 plants were refreshed weekly.

394 Furthermore, we compared the evolved individual performance of mites from a control

395

396

397

398

399

400

401

402

403

population (maintained on the initial host plant) with mites adapting to cucumber with or without

the ghost competitor. We found that individuals from populations of T. urticae grown on cucumber plants without $T$. ludeni reached a higher evolved fecundity on the novel host plant

than individuals from the control population (maintained on the initial host plant), implying local adaptation to the novel host (Fig. 3b). This difference in evolved individual fecundity with the control population was not found for individuals from the populations that were initially under competition with $T$. ludeni when considering all these populations as a single group (so not splitting them according to initial population size of $T$. ludeni). However, the fecundity of this group under interspecific competition was also not significantly different from the adapted $T$. 
404 urticae populations without interspecific competition, indicating that this group was intermediate

405 between the adapted group without interspecific competition and the control population.

406 We explain this by the various degrees of early competitive pressure in this group under

407 heterospecific competition, the main result of our study. We suggest that not finding a difference

408 in fecundity with the control population may be due to an initial trade-off between performance

409 on the novel host and the ability to compete with heterospecifics (Siepielski et al., 2016). It is

410 possible that the populations under competition initially adapted to the competitor and that this

411 lowered the amount of standing genetic variation necessary for adaptation to the novel host. A

412 likely alternative explanation for these various degrees of early competitive pressure is variation

413 in drift effects which is more probable in the populations under heterospecific competition

414 because of the lower initial population size per species. Under a scenario of strong drift effects

415 we would expect large differences in performance between replicates with and without T. ludeni

416 (and thus with high or low initial population size for T. urticae), but they were quite similar (Fig.

417 S1), so strong drift can be largely discarded as a driver of the observed evolutionary dynamics.

Individuals from populations where half of the population was replaced with heterospecifics could not adapt as well to the novel host plants as the populations consisting of only conspecifics. This could be due to a higher selection pressure from conspecifics compared to heterospecifics which may in turn be due to the larger initial population sizes or due to a larger niche overlap (Bolnick, 2001; Svanbäck \& Bolnick, 2007).

425 The history of species in a community can have an impact on interspecific interactions (Fukami, 426 2015). The magnitudes of such historical contingencies do, however, strongly differ among 
427 species and environments (Vannette \& Fukami, 2014). Differences in historical contingency may

428 explain why some populations experience radiations, whereas others from the same clade do not

429 achieve this under seemingly similar conditions (Seehausen, 2007). Our results suggest that

430 increased interspecific competition leads to higher selection pressures and thus improved

431 performance (Fig. 3b). Our results coincide in this respect with other empirical work

432 demonstrating that increased competition with heterospecifics increased local adaptation in

433 bunchgrasses (Rice \& Knapp, 2008). Similarly, intraguild predation between lizard species

434 increased the selection pressure and led to strong divergence in morphological adaptation as

435 associated with niche specialisation (Stuart et al., 2014).

436

437 Nevertheless, we have to be careful with generalising our results. First, we chose small

438 populations sizes as they are more biologically relevant, but this may limit adaptation and

439 establishment (Del Castillo et al., 2011; Yates \& Fraser, 2014). We also used populations that

440 have been maintained in the lab for many generations, probably leading to a decrease in genetic

441 variation compared to wild populations. The problems we encountered in creating isofemale

442 lines for T. ludeni could be an indication of inbreeding depression. However, we are confident

443 that our results are robust as we could still provide evidence for local adaptation in the

444 populations of T. urticae without competitor (Fig. 3b). This suggests that the initial amount of

445 genetic variation did not limit T. urticae in our study.

446 Second, it is impossible to add a competitor without changing total population sizes, population

447 densities, or island sizes; all of these affect genetic variation and drift (Del Castillo et al., 2011;

448 Alzate, Etienne \& Bonte, 2019). As it is known that larger populations usually contain more

449 genetic variation, we chose to standardise this by means of isofemale lines, knowing that this 
450 might create differences in drift among treatments. One way to better disentangle the effects of

451 drift from those of selection with our small population size would have been to increase the

452 number of replicates which was difficult for logistical reasons.

453 Third, our experimental design is not strictly suitable to assess adaptation in the interspecific

454 competition treatment, as we did not keep a heterospecific control population on bean (again for

455 logistical reasons). Hence, we cannot disentangle the effect of changes in fecundity due to

456 competition (independent of the novel environment) from the effect of competition on adaptation

457 to the novel environment. Although this means that we cannot detect adaptation in the treatment

458 under ghost competition, we did find a positive influence of the density of the ghost competitor

459 on fecundity, meaning that early competitive pressures substantially matter and providing

460 evidence for eco-evolutionary dynamics. 


\section{CONCLUSIONS}

462 In conclusion, we have shown the importance of early selection pressures such as ghost

463 competition. Even when one species becomes extinct, the competition signature continues to

464 affect the adaptation process of the successful species. 


\section{ACKNOWLEDGEMENTS}

466 We thank Viki Vandomme, Angelica Alcantara, Pieter Vantieghem, Katrien Van Petegem,

467 Stefano Masier, Matti Pisman, Mike Creutz, Hilde De Nil and Johan Bisschop for helping during

468 the research experiments, and to Sarah Magalhães for providing the strains of T. ludeni. We

469 thank the Terrestrial Ecology department of Ghent University and the Centre for Ecology,

470 Evolution and Environmental Changes of the University of Lisbon for the spider mite

471 populations. 
472 REFERENCES

473 Alzate A, Bisschop K, Etienne RS, Bonte D. 2017. Interspecific competition counteracts

474 negative effects of dispersal on adaptation of an arthropod herbivore to a new host. Journal

475 of Evolutionary Biology 30:1966-1977. DOI: 10.1111/jeb.13123.

476

Alzate A, Etienne RS, Bonte D. 2019. Experimental island biogeography demonstrates the importance of island size and dispersal for the adaptation to novel habitats. Global Ecol Biogeogr 28:238-247. DOI: 10.1111/geb.12846.

Bartoń KA. 2019.MuMIn: multi-model inference. R package version 1.43.6. Available at https://cran.r-project.org/package $=$ MuMIn

481

482

483

484

485

486

487

488

489

490

491

492

493

494

Bengtsson J. 1989. Interspecific competition increases local extinction rate in a metapopulation system. Nature 340:713-715. DOI: 10.1038/340713a0.

Bisschop K, Mortier F, Etienne RS, Bonte D. 2019. Transient local adaptation and source-sink dynamics in experimental populations experiencing spatially heterogeneous environments. Proceedings of the Royal Society B: Biological Sciences 286:20190738. DOI: 10.1098/rspb.2019.0738.

Bitume E V., Bonte D, Ronce O, Bach F, Flaven E, Olivieri I, Nieberding CM. 2013. Density and genetic relatedness increase dispersal distance in a subsocial organism. Ecology Letters 16:430-437. DOI: 10.1111/ele.12057.

Bolnick DI. 2001. Intraspecific competition favours niche width expansion in Drosophila melanogaster. Nature 410:463-466. DOI: 10.1038/35068555.

Bonte D, De Roissart A, Vandegehuchte ML, Ballhorn DJ, van Leeuwen T, de la Peña E. 2010. Local adaptation of aboveground herbivores towards plant phenotypes induced by soil biota. PLoS ONE 5:e11174. DOI: 10.1371/journal.pone.0011174. 
495 496

497 498

499

500

501

502

503

504

505

506

507

508

509

510

511

512

513

514

515

516

517

Bonte D, De Roissart A, Wybouw N, Van Leeuwen T. 2014. Fitness maximization by dispersal: evidence from an invasion experiment. Ecology 95:3104-3111. DOI: 10.1890/13-2269.1.

Brooks ME, Kristensen K, van Benthem KJ, Magnusson A, Berg CW, Nielsen A, Skaug HJ, Mächler M, Bolker BM. 2017. glmmTMB balances speed and flexibility among packages for zero-inflated generalized linear mixed modeling. The R Journal 9:378-400. DOI: 10.3929/ETHZ-B-000240890.

Del Castillo RF, Trujillo-Argueta S, Sánchez-Vargas N, Newton AC. 2011. Genetic factors associated with population size may increase extinction risks and decrease colonization potential in a keystone tropical pine. Evolutionary Applications 4:574-588. DOI: 10.1111/j.1752-4571.2010.00177.x.

Chesson P. 2000. Mechanisms of maintenance of species diversity. :343-366.

Dahirel M, Masier S, Renault D, Bonte D. 2019. The distinct phenotypic signatures of dispersal and stress in an arthropod model: from physiology to life history. The Journal of experimental biology 222:jeb203596. DOI: 10.1242/jeb.203596.

Delignette-Muller ML. 2015. fitdistrplus: an R package for fitting distributions. Journal of Statistical Software 64:1-34. DOI: 10.18637/jss.v064.i04.

Dermauw W, Pym A, Bass C, Van Leeuwen T, Feyereisen R. 2018. Does host plant adaptation lead to pesticide resistance in generalist herbivores? Current Opinion in Insect Science 26:25-33. DOI: 10.1016/j.cois.2018.01.001.

Després L, David JP, Gallet C. 2007. The evolutionary ecology of insect resistance to plant chemicals. Trends in Ecology and Evolution 22:298-307. DOI: 10.1016/j.tree.2007.02.010.

Diamond J, Pimm SL, Gilpin ME, LeCroy M. 1989. Rapid evolution of character displacement in Myzomelid honeyeaters. The American Naturalist 134:675-708. DOI: 10.1086/285006. 
518 Fry JD. 1989. Evolutionary adaptation to host plants in a laboratory population of the

519 phytophagous mite Tetranychus urticae Koch. Oecologia 81:559-565. DOI:

$520 \quad 10.1007 / B F 00378969$.

521 Fukami T. 2015. Historical contingency in community assembly: integrating niches, species

522 pools, and priority effects. Annual Review of Ecology, Evolution, and Systematics 46:1-23.

523 DOI: 10.1146/annurev-ecolsys-110411-160340.

524 Godinho DP, Janssen A, Dias T, Cruz C, Magalhães S. 2016. Down-regulation of plant defence

525 in a resident spider mite species and its effect upon con- and heterospecifics. Oecologia

526 180:161-167. DOI: 10.1007/s00442-015-3434-z.

527 Gould F. 1979. Rapid host range evolution in a population of the phytophagous mite Tetranychus

528 urticae Koch. Evolution 33:791-802. DOI: 10.1111/j.1558-5646.1979.tb04735.x.

529 Griffis MR, Jaeger RG. 1998. Competition leads to an extinction-prone species of salamander:

530 Interspecific territoriality in a metapopulation. Ecology 79:2494-2502. DOI: 10.1890/0012-

531 9658(1998)079[2494:CLTAEP]2.0.CO;2.

532 Hardin JW, Hilbe JM. 2007. Generalized Linear Models and Extensions. Texas: Stata Press.

533 Hawkes C V., Keitt TH. 2015. Resilience vs. historical contingency in microbial responses to 534 environmental change. Ecology Letters 18:612-625. DOI: 10.1111/ele.12451.

535 Holmes EE, Wilson HB. 1998. Running from trouble: long-distance dispersal and the 536 competitive coexistence of inferior species. The American Naturalist 151:578-586. DOI:

$537 \quad 10.1086 / 286143$.

538 Holt RD. 1987. On the relation between niche overlap and competition: the effect of

539 incommensurable niche dimensions. Oikos 48:110-114.

540 Hutchinson GE. 1957. Concluding Remarks. Cold Spring Harbor Symposia on Quantitative 
Biology 22:415-427. DOI: 10.1101/sqb.1957.022.01.039.

542 Jaeger RG. 1970. Potential extinction through competition between two species of terrestrial 543 salamanders. Evolution 24:632-642. DOI: 10.1111/j.1558-5646.1970.tb01797.x.

544 Kant MR, Jonckheere W, Knegt B, Lemos F, Liu J, Schimmel BCJ, Villarroel CA, Ataide LMS, 545 Dermauw W, Glas JJ, Egas M, Janssen A, Van Leeuwen T, Schuurink RC, Sabelis MW, 546 Alba JM. 2015. Mechanisms and ecological consequences of plant defence induction and 547 suppression in herbivore communities. Annals of Botany 115:1015-1051. DOI: 10.1093/aob/mcv054.

549 Kawecki TJ, Lenski RE, Ebert D, Hollis B, Olivieri I, Whitlock MC. 2012. Experimental 550 evolution. Trends in Ecology and Evolution 27:547-560. DOI: 10.1016/j.tree.2012.06.001.

551 Klopfer PH, MacArthur RH. 1961. On the causes of tropical species diversity: niche overlap. $552 \quad$ DOI: $10.1086 / 282179$.

553

554

555

556

557

558

559

560

561

562

563

Lankau RA. 2011. Rapid evolutionary change and the coexistence of species. Annual Review of Ecology, Evolution, and Systematics 42:335-54. DOI: 10.1146/annurev-ecolsys-102710145100.

Law R, Daniel Morton R. 1996. Permanence and the assembly of ecological communities. Ecology 77:762-775. DOI: 10.2307/2265500.

Lawrence D, Fiegna F, Behrends V, Bundy JG, Phillimore AB, Bell T, Barraclough TG. 2012. Species interactions alter evolutionary responses to a novel environment. PLoS Biology 10:e1001330. DOI: 10.1371/journal.pbio.1001330.

Van Leeuwen T, Stillatus V, Tirry L. 2004. Genetic analysis and cross-resistance spectrum of a laboratory-selected chlorfenapyr resistant strain of two-spotted spider mite (Acari: Tetranychidae). Experimental and Applied Acarology 32:249-261. DOI: 
565

566

567

568

569

570

571

572

573

574

575

576

577

578

579

580

581

582

583

584

585

586

Lenth R. 2019.emmeans: estimated marginal means, aka least-squares means. R package version

\subsubsection{Available at https://cran.r-project.org/package=emmeans}

Li J, Margolies DC. 1993. Effects of mite age, mite density, and host quality on aerial dispersal behavior in the twospotted spider mite. Entomologia Experimentalis et Applicata 68:79-86. DOI: 10.1111/j.1570-7458.1993.tb01691.x.

Lindsey HA, Gallie J, Taylor S, Kerr B. 2013. Evolutionary rescue from extinction is contingent on a lower rate of environmental change. Nature 494:463-467. DOI: $10.1038 /$ nature 11879 .

Magalhães S, Blanchet E, Egas M, Olivieri I. 2009. Are adaptation costs necessary to build up a local adaptation pattern? BMC Evolutionary Biology 9:182. DOI: 10.1186/1471-2148-9182.

Magalhães S, Blanchet E, Egas M, Olivieri I. 2011. Environmental effects on the detection of adaptation. Journal of Evolutionary Biology 24:2653-2662. DOI: 10.1111/j.14209101.2011.02388.x.

Magalhães S, Fayard J, Janssen A, Carbonell D, Olivieri I. 2007. Adaptation in a spider mite population after long-term evolution on a single host plant. Journal of Evolutionary Biology 20:2016-2027. DOI: 10.1111/j.1420-9101.2007.01365.x.

Magnusson A, Skaug H, Nielsen A, Berg CW, Kristensen K, Maechler M, van Bentham K, Bolker BM, Brooks ME. 2018.Troubleshooting with glmmTMB. Available at https://cran.r-project.org/web/packages/glmmTMB/vignettes/troubleshooting.html

Mallon CA, Le Roux X, Van Doorn GS, Dini-Andreote F, Poly F, Salles JF. 2018. The impact of failure: unsuccessful bacterial invasions steer the soil microbial community away from the invader's niche. ISME Journal 12:728-741. DOI: 10.1038/s41396-017-0003-y. 
587 Miller TE, TerHorst CP, Burns JH. 2009. The ghost of competition present. The American $588 \quad$ Naturalist 173:347-353. DOI: 10.1086/596531.

589 Osmond MM, de Mazancourt C. 2013. How competition affects evolutionary rescue.

$590 \quad$ Philosophical transactions of the Royal Society of London B, Biological sciences

$591 \quad 368: 20120085$. DOI: 10.1098/rstb.2012.0085.

592 Van Petegem K, Moerman F, Dahirel M, Fronhofer EA, Vandegehuchte ML, Van Leeuwen T, 593 Wybouw N, Stoks R, Bonte D. 2018. Kin competition accelerates experimental range 594 expansion in an arthropod herbivore. Ecology Letters 21:225-234. DOI: 10.1111/ele.12887. 595 Reznick DN, Ghalambor CK. 2001. The population ecology of contemporary adaptations: what 596 empirical studies reveal about the conditions that promote adaptive evolution. Genetica 597 598 112:183-198. DOI: 10.1023/A:1013352109042.

Rice KJ, Knapp EE. 2008. Effects of competition and life history stage on the expression of local adaptation in two native bunchgrasses. Restoration Ecology 16:12-23. DOI:

601

602

603

604

605

606 10.1111/j.1526-100X.2007.00257.x.

Des Roches S, Post DM, Turley NE, Bailey JK, Hendry AP, Kinnison MT, Schweitzer JA, Palkovacs EP. 2018. The ecological importance of intraspecific variation. Nature Ecology and Evolution 2:57-64. DOI: 10.1038/s41559-017-0402-5.

Sarmento RA, Lemos F, Bleeker PM, Schuurink RC, Pallini A, Oliveira MGA, Lima ER, Kant M, Sabelis MW, Janssen A. 2011. A herbivore that manipulates plant defence. Ecology

607 Schluter D. 1994. Experimental evidence that competition promotes divergence in adaptive Letters 14:229-236. DOI: 10.1111/j.1461-0248.2010.01575.x. 608 radiation. Science 266:798-801. DOI: 10.1126/science.266.5186.798.

609 Seehausen O. 2007. Evolution and ecological theory: chance, historical contingency and 
610 ecological determinism jointly determine the rate of adaptive radiation. Heredity 99:361611 363. DOI: 10.1038/sj.hdy.6801047.

612 Siepielski AM, Nemirov A, Cattivera M, Nickerson A. 2016. Experimental evidence for an eco613 evolutionary coupling between local adaptation and intraspecific competition. The 614 American Naturalist 187:447-456. DOI: 10.1086/685295.

615 Silvertown J. 2004. The ghost of competition past in the phylogeny of island endemic plants. 616 Journal of Ecology 92:168-173. DOI: 10.1111/j.1365-2745.2004.00853.x.

617 Stuart YE, Campbell TS, Hohenlohe PA, Reynolds RG, Revell LJ, Losos JB. 2014. Rapid 618 evolution of a native species following invasion by a congener. Science 346:463-466. DOI: $10.1126 /$ science. 1257008 .

620

621

622

623

624

625

626

627

628

629 630

631

632

Svanbäck R, Bolnick DI. 2007. Intraspecific competition drives increased resource use diversity within a natural population. Proceedings of the Royal Society B: Biological Sciences 274:839-844. DOI: 10.1098/rspb.2006.0198.

Turcotte MM, Reznick DN, Hare JD. 2011. The impact of rapid evolution on population dynamics in the wild: experimental test of eco-evolutionary dynamics. Ecology Letters 14:1084-1092. DOI: 10.1111/j.1461-0248.2011.01676.x.

Turcotte MM, Reznick DN, Hare JD. 2013. Experimental Test of an Eco-Evolutionary Dynamic Feedback Loop between Evolution and Population Density in the Green Peach Aphid. The American Naturalist 181:S46-S57. DOI: 10.1086/668078.

Vannette RL, Fukami T. 2014. Historical contingency in species interactions: towards nichebased predictions. Ecology Letters 17:115-124. DOI: 10.1111/ele.12204.

Yates MC, Fraser DJ. 2014. Does source population size affect performance in new environments? Evolutionary Applications 7:871-882. DOI: 10.1111/eva.12181. 
633 Zhang ZQ. 2003. Mites of greenhouses: identification, biology and control. Wallingford: CABI 634 Publishing. DOI: 10.1079/9780851995908.0000.

635 Zhang P-J, Zheng S-J, van Loon JJA, Boland W, David A, Mumm R, Dicke M. 2009. Whiteflies 636 interfere with indirect plant defense against spider mites in Lima bean. Proceedings of the 637 National Academy of Sciences 106:21202-21207. DOI: 10.1073/pnas.0907890106.

638 Zhao XF, Buckling A, Zhang QG, Hesse E. 2018. Specific adaptation to strong competitors can 639 offset the negative effects of population size reductions. Proceedings of the Royal Society 640 B: Biological Sciences 285:20180007. DOI: 10.1098/rspb.2018.0007. 


\section{Figure 1: The experimental set-up.}

642 Adult females from 13 inbred lines of $T$. urticae $(T u)$ were equally divided over the different

643 treatments to create the same starting genetic variation. Populations with $T$. ludeni (Tl) had a

644 higher genetic variation as only six inbred lines were used and supplemented with the stock

645 population. The treatments were a control population on bean plants (yellow box for $T u$ and

646 white for $T l$ ), a competition treatment with both species present on cucumber (dark green or dark

647 pink box for $T u$ and $T l$ respectively) or a no competition treatment on cucumber (light green box

648 for $T u$ and light pink box for $T l$ ). The density of the populations of mites on cucumber was

649 tracked for ecological dynamics and individual fecundity tests were performed on the novel and

650 initial host plants after two generations on the initial host plant for homogenising maternal

651 effects. The boxes have the same colours as used in Figs. 2 and 3.

652

653 Figure 2: The dynamics and performance of the ghost competitor.

654 (A) Overview of the population density for the different treatments of the experimental

655 populations on cucumber. Population density of Tetranychus urticae (green dots) and T. ludeni

656 (pink dots) measured as the sum of the abaxial and adaxial density (number of adult

657 females $/ \mathrm{cm}^{2}$ ) per island through time. The lighter colours correspond to the populations in

658 absence of the competing species and the darker to the treatment where both species are present.

659 The lines are smoothing curves with their respective 95\% confidence interval. (B) Comparison of

660 the densities for the different treatments at the plateau phase (starting from 200 days). The letters

661 above the violin plots indicate the significant differences. No significant difference was found

662 between population densities of T. urticae with or without initial competition, and both

663 populations reached significantly higher densities than $T$. ludeni. The violin plots show the 
664 observed data, and the points and lines show the mean model estimates and their 95\% confidence

665 interval, respectively. (C) Comparison of the individual performance of the control populations

666 of T. ludeni and T. urticae (both maintained on bean plants) on bean and cucumber leaf discs at

667 the first measured time point. The fecundity of $T$. ludeni is significantly lower than that of $T$.

668 urticae, on both bean and cucumber.

669

670 Figure 3: Fecundity affected by ghost competition.

671 On the x-axis the different treatments (the control population of T. urticae from bean (yellow), $T$.

672 urticae with ghost competition of T. ludeni (dark green), and T. urticae from cucumber but

673 without T. ludeni (light green)) are presented. The scale on the x-axis indicates the early

674 competitive pressure of $T$. ludeni (average number of adult females $/ \mathrm{cm}^{2}$ during the first month)

675 for the replicates of the treatment under ghost competition; this treatment is shown in the grey

676 box. On the y-axis the fecundity (number of eggs after six days) of T. urticae is presented. The

677 variable time was not present in the best-fitting model, so we presented all data points per

678 treatment independent of the time it was measured. The plot on the left (A) shows the fecundity

679 assessed on bean, while the one on the right (B) gives the results assessed on cucumber.

680 Populations without T. ludeni maintained on cucumber (light green) performed significantly

681 better on cucumber than the control (yellow) and seemed therefore locally adapted. The

682 treatment under competition (dark green) was intermediate between both other treatments. A

683 significant relationship between the density of T. ludeni (in the treatment under ghost

684 competition; dark green) and the fecundity of T. urticae was found when assessed on cucumber.

685 This indicates that early experienced selection pressures can exert a persistent evolutionary

686 signal on species' performance in novel environments. Each violin plot presents the observed 
687 data, while the points and lines show the means of the model estimate and their $95 \%$ confidence

688 interval, respectively.

689

690

691

Table 1: Model selection.

692 Overview of the best models based on the lowest AICc with an AICc weight of at least 0.100.

693 Abbreviations of fixed variables in maximal model: fecundity (fec.), dens. Tu (initial density $\mathrm{T}$.

694 urticae), dens. T1 (initial density T. ludeni), t (time), Tu comp./no comp. (T. urticae with

695 competition/without competition), Tl no comp. (T. ludeni without competition), and treat.

696 (treatment).

697

698

Table 2: Chi-square statistics for the maximal models before model selection. The results for 699 the Wald Chi-square tests are presented for the maximal models.

700

701

Table 3: Pairwise comparisons adjusted for multiple comparisons (Tukey method). The

702 estimates provided in the table are the raw and untransformed estimates (negative binomial distribution). The estimates are the differences in fecundity for A. and C. and in density for B.

704

705

Table 4: Summary of the final best-fitting GLMM explaining reproductive performance.

706

The values provided in the table are the raw and untransformed estimates due to the negative

707 binomial distribution in the model.

708

Table 5: Model selection (A) and Wald $\chi^{2}$ test (B) for the influence of total initial density on 


\section{Figure 1}

The experimental set-up

Adult females from 13 inbred lines of $T$. urticae $(T u)$ were equally divided over the different treatments to create the same starting genetic variation. Populations with $T$. ludeni $(T I)$ had a higher genetic variation as only six inbred lines were used and supplemented with the stock population. The treatments were a control population on bean plants (yellow box for Tu and white for $T I$ ), a competition treatment with both species present on cucumber (dark green or dark pink box for $T u$ and $T I$ respectively) or a no competition treatment on cucumber (light green box for $T u$ and light pink box for $T I)$. The density of the populations of mites on cucumber was tracked for ecological dynamics and individual fecundity tests were performed on the novel and initial host plants after two generations on the initial host plant for homogenising maternal effects. The boxes have the same colours as used in Figs. 2 and 3.

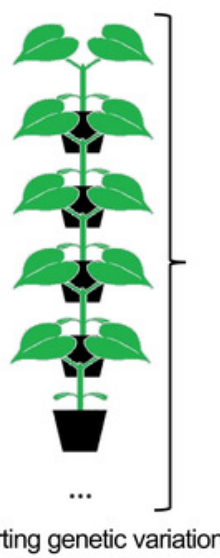

13 inbred lines of T. urticae

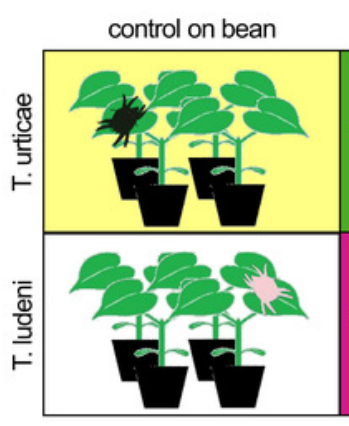

selection on cucumber in experimental evolution for ten months ( 25 generations) tracking density of mites on cucumber for ecological dynamics

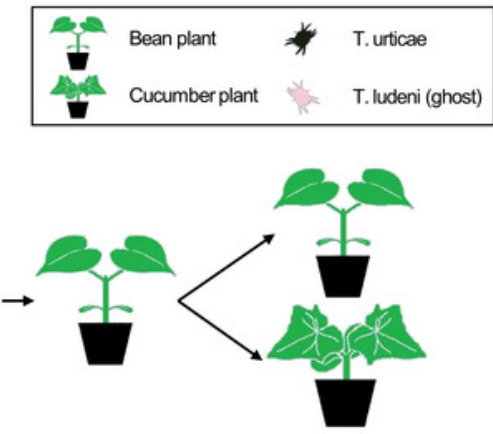

homogenising maternal effects

fecundity test measuring eggs on different host plants as proxy for adaptation 


\section{Figure 2}

The dynamics and performance of the ghost competitor

(A) Overview of the population density for the different treatments of the experimental populations on cucumber. Population density of Tetranychus urticae (green dots) and $T$. Iudeni (pink dots) measured as the sum of the abaxial and adaxial density (number of adult females $/ \mathrm{cm}^{2}$ ) per island through time. The lighter colours correspond to the populations in absence of the competing species and the darker to the treatment where both species are present. The lines are smoothing curves with their respective $95 \%$ confidence interval. (B) Comparison of the densities for the different treatments at the plateau phase (starting from 200 days). The letters above the violin plots indicate the significant differences. No significant difference was found between population densities of $T$. urticae with or without initial competition, and both populations reached significantly higher densities than $T$. Iudeni. The violin plots show the observed data, and the points and lines show the mean model estimates and their $95 \%$ confidence interval, respectively. (C) Comparison of the individual performance of the control populations of $T$. ludeni and $T$. urticae (both maintained on bean plants) on bean and cucumber leaf discs at the first measured time point. The fecundity of $T$. ludeni is significantly lower than that of $T$. urticae, on both bean and cucumber. 

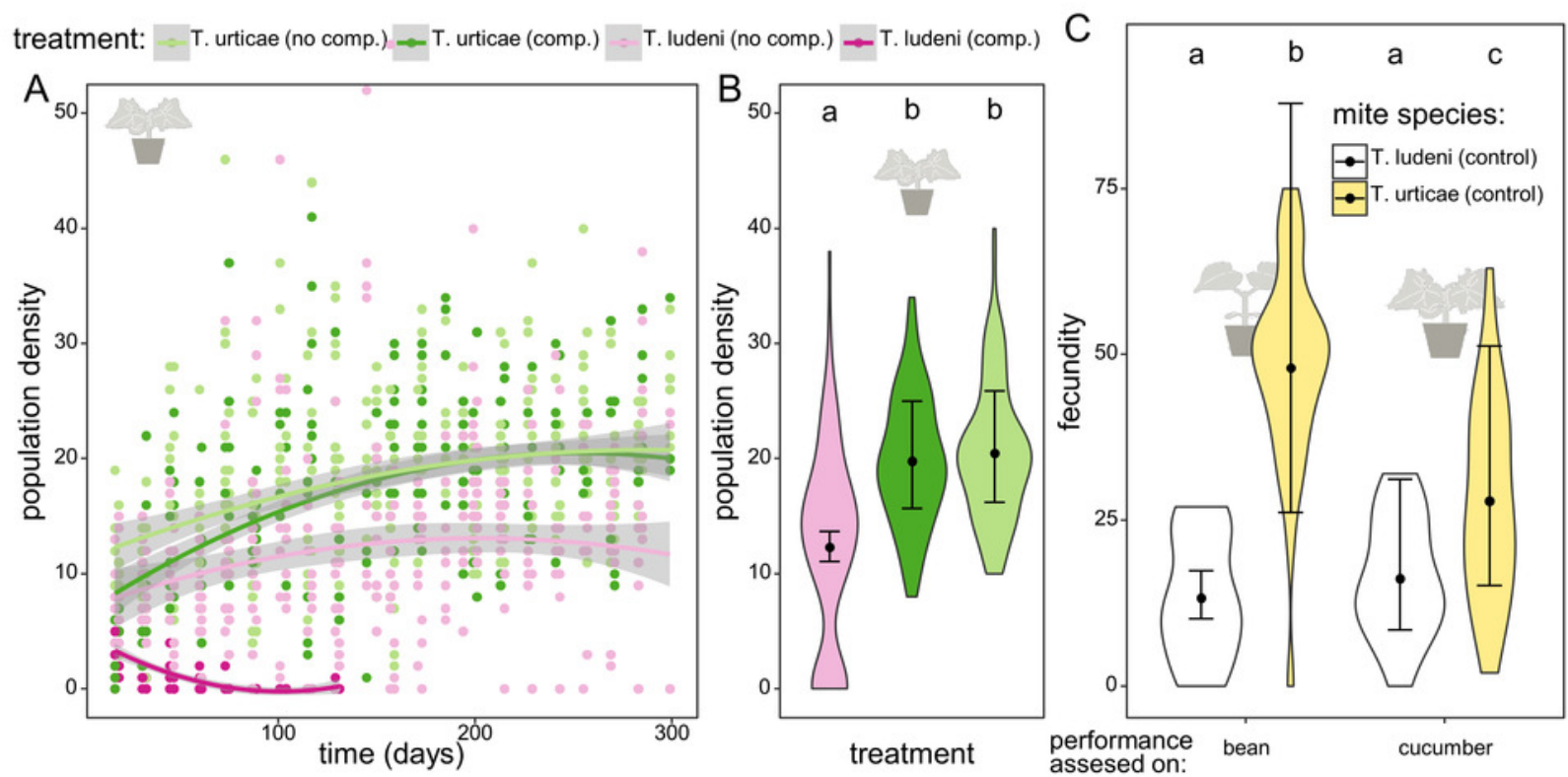


\section{Figure 3}

Fecundity affected by ghost competition

On the $x$-axis the different treatments (the control population of $T$. urticae from bean (yellow), T. urticae with ghost competition of T. ludeni (dark green), and T. urticae from cucumber but without $T$. ludeni (light green)) are presented. The scale on the $x$-axis indicates the early competitive pressure of $T$. ludeni (average number of adult females $/ \mathrm{cm}^{2}$ during the first month) for the replicates of the treatment under ghost competition; this treatment is shown in the grey box. On the y-axis the fecundity (number of eggs after six days) of $T$. urticae is presented. The variable time was not present in the best-fitting model, so we presented all data points per treatment independent of the time it was measured. The plot on the left (A) shows the fecundity assessed on bean, while the one on the right (B) gives the results assessed on cucumber. Populations without $T$. Iudeni maintained on cucumber (light green) performed significantly better on cucumber than the control (yellow) and seemed therefore locally adapted. The treatment under competition (dark green) was intermediate between both other treatments. A significant relationship between the density of $T$. Iudeni (in the treatment under ghost competition; dark green) and the fecundity of $T$. urticae was found when assessed on cucumber. This indicates that early experienced selection pressures can exert a persistent evolutionary signal on species' performance in novel environments. Each violin plot presents the observed data, while the points and lines show the means of the model estimate and their $95 \%$ confidence interval, respectively. 

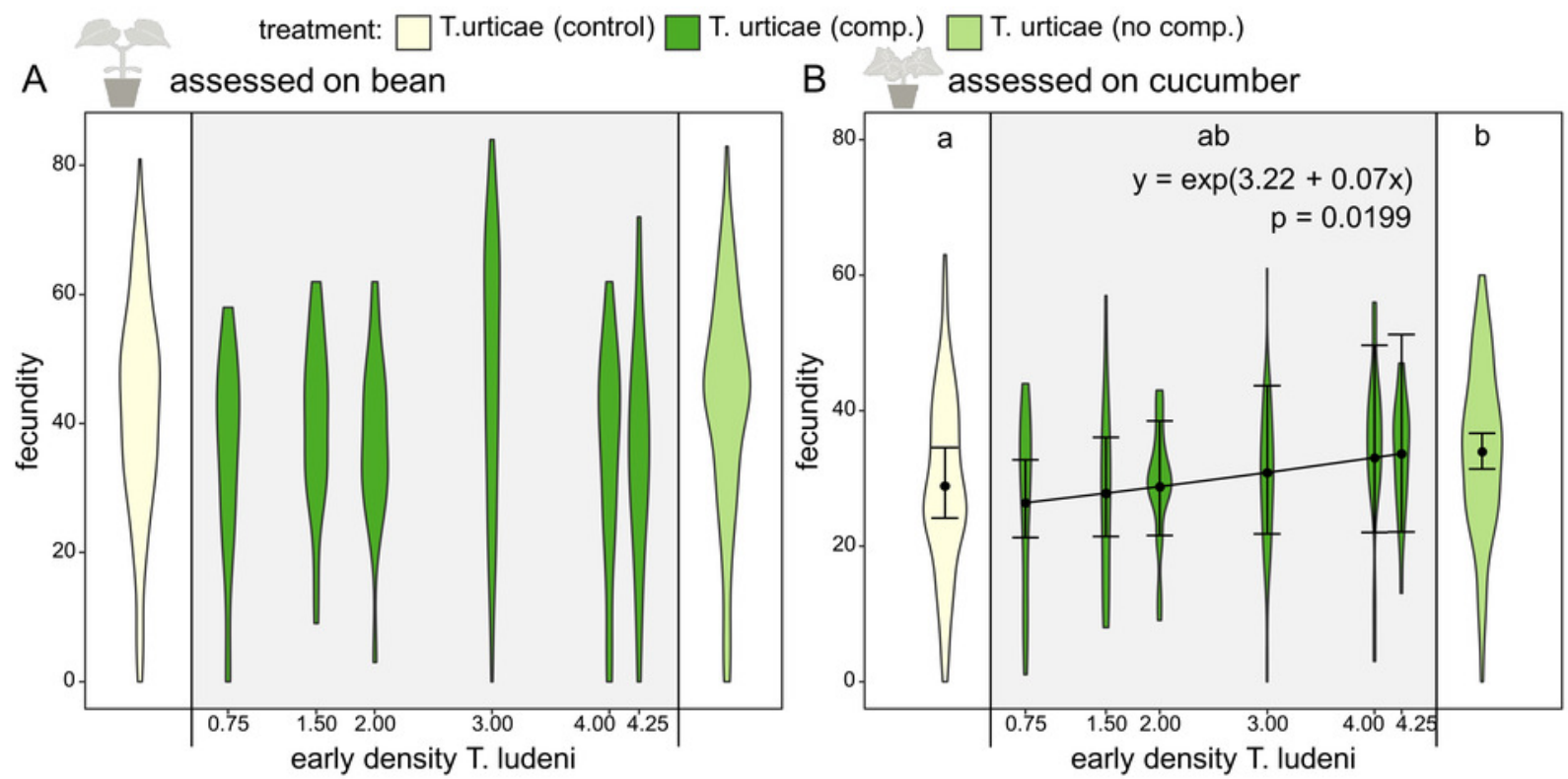


\section{Table $\mathbf{1}$ (on next page)}

Model selection

Overview of the best models based on the lowest AICC with an AICC weight of at least 0.100. Abbreviations of fixed variables in maximal model: fecundity (fec.), dens. Tu (initial density T. urticae), dens. TI (initial density T. ludeni), t (time), Tu comp./no comp. (T. urticae with competition/without competition), TI no comp. (T. ludeni without competition), and treat. (treatment). 


\section{Table 1: Model selection.}

2 Overview of the best models based on the lowest AICc with an AICc weight of at least 0.100.

3 Abbreviations of fixed variables in maximal model: fecundity (fec.), dens. $T u$ (initial density $T$.

4 urticae), dens. $T l$ (initial density $T$. ludeni), t (time), Tu comp./no comp. (T. urticae with

5 competition/without competition), $T l$ no comp. ( $T$. ludeni without competition), and treat.

6 (treatment).

\section{Model}

df LogLik AICc $\triangle$ AICc AICc weight

The dynamics and performance of the ghost competitor

Max. model: fec. $\sim$ plant species * mite species $(\mathrm{df}=5)$

Plant species * mite species

$\begin{array}{llll}5 & -415.037 & 840.7 & 0.00\end{array}$

0.948

\section{Signature of the ghost competitor on performance of $T$. urticae}

Fecundity assessed on bean

Max. model: fec. $\sim \mathrm{t}+$ dens $T u+$ dens $T l+\mathrm{t}:$ dens. $T u+\mathrm{t}:$ dens. $T l+(1 \mid$ block$/$ island $)(\mathrm{df}=18)$

$\begin{array}{llllll}\text { No fixed effects } & 4 & -768.410 & 1545.1 & 0.00 & 0.237 \\ \text { Time } & 8 & -764.312 & 1545.5 & 0.45 & 0.190 \\ \text { Initial density } T \text {. urticae } & 5 & -767.785 & 1545.9 & 0.87 & 0.153\end{array}$

Fecundity assessed on cucumber

Max. model: fec. $\sim \mathrm{t}+$ dens. $T u+$ dens. $T l+\mathrm{t}:$ dens. $T u+\mathrm{t}:$ dens. $T l+(1$ island $)(\mathrm{df}=17)$

$\begin{array}{llllll}\text { Initial density } T \text {. ludeni } & 4 & -594.802 & 1197.9 & 0.00 & 0.484 \\ \text { Initial density } T \text {. urticae + init. dens. } T l & 5 & -594.602 & 1199.6 & 1.74 & 0.203 \\ \text { Initial density } T \text {. urticae } & 4 & -596.243 & 1200.8 & 2.88 & 0.115\end{array}$

Demography from plateau phase

Max. model: dens. $\sim$ treat. (Tu comp./no comp., $T l$ no comp. $)+(1 \mid \mathrm{t})+(1 \mid$ block/island $)(\mathrm{df}=7)$ Treatment

$\begin{array}{llll}7 & -1185.931 & 2386.2 & 0.00\end{array}$

Performance of $T$. urticae

Max. model: fec. $\sim$ treat. (Tu control/Tu comp./Tu no comp.) $* \mathrm{t}+(1 \mid$ block/island $)(\mathrm{df}=18)$

\begin{tabular}{llllll} 
Treatment & 6 & -1846.641 & 3705.5 & 0.00 & 0.751 \\
No fixed effects & 4 & -1849.890 & 3707.9 & 2.40 & 0.226 \\
\hline
\end{tabular}




\section{Table 2 (on next page)}

Chi-square statistics for the maximal models before model selection

The results for the Wald Chi-square tests are presented for the maximal models. 
1 Table 2: Chi-square statistics for the maximal models before model selection. The results for 2 the Wald Chi-square tests are presented for the maximal models.

Independent variables Chisq

Df $\quad \operatorname{Pr}(>$ Chisq $)$

\begin{tabular}{|c|c|c|c|c|c|}
\hline \multicolumn{6}{|c|}{ The dynamics and performance of the ghost competitor } \\
\hline & Plant species & 5.1851 & 1 & 0.0228 & $*$ \\
\hline & Mite species & 56.1019 & 1 & $6.881 \mathrm{e}-14$ & $* * *$ \\
\hline & Plant species : mite species & 9.0064 & 1 & 0.0027 & $* *$ \\
\hline \multicolumn{6}{|c|}{ Signature of the ghost competitor on performance of $T$. urticae } \\
\hline \multirow[t]{5}{*}{ Fecundity on bean } & Time & 8.4890 & 4 & 0.0752 & $\cdot$ \\
\hline & Initial density $T$. ludeni & 0.8919 & 1 & 0.3450 & \\
\hline & Initial density $T$. urticae & 1.4948 & 1 & 0.2215 & \\
\hline & Time : init. dens. $T l$ & 1.3088 & 4 & 0.8599 & \\
\hline & Time : init. dens. $T u$ & 4.5696 & 4 & 0.3344 & \\
\hline \multirow{5}{*}{$\begin{array}{l}\text { Fecundity on } \\
\text { cucumber }\end{array}$} & Time & 4.6468 & 4 & 0.3255 & \\
\hline & Initial density $T$. ludeni & 4.1050 & 1 & 0.0428 & $*$ \\
\hline & Initial density $T$. urticae & 0.6019 & 1 & 0.4379 & \\
\hline & Time : init. dens. $T l$ & 1.7385 & 4 & 0.7837 & \\
\hline & Time : init. dens. $T u$ & 4.0678 & 4 & 0.3969 & \\
\hline $\begin{array}{l}\text { Demography (from } \\
\text { plateau phase) }\end{array}$ & Treatment & 74.1960 & 2 & $<2.2 \mathrm{e}-16$ & $* * *$ \\
\hline \multicolumn{6}{|c|}{ Performance of $T$. urticae } \\
\hline & Treatment & 9.8340 & 2 & 0.0073 & $* *$ \\
\hline & Time & 0.8189 & 4 & 0.9359 & \\
\hline & Treatment : time & 7.9751 & 8 & 0.4359 & \\
\hline
\end{tabular}

3 


\section{Table 3(on next page)}

Pairwise comparisons adjusted for multiple comparisons (Tukey method)

The estimates provided in the table are the raw and untransformed estimates (negative binomial distribution). The estimates are the differences in fecundity for $A$. and $C$. and in density for B. 
1 Table 3: Pairwise comparisons adjusted for multiple comparisons (Tukey method). The

2 estimates provided in the table are the raw and untransformed estimates (negative binomial

3 distribution). The estimates are the differences in fecundity for A. and C. and in density for B.

\begin{tabular}{|c|c|c|c|c|c|c|}
\hline Contrast & Estimate & SE & $\mathrm{df}$ & t ratio & $\mathrm{p}$ value & \\
\hline \multirow{2}{*}{\multicolumn{7}{|c|}{$\begin{array}{l}\text { A. The dynamics and performance of the ghost competitor } \\
\text { Comparison control population } T u \text { and } T l \text { on bean and cucumber (at first measured time point) }\end{array}$}} \\
\hline & & & & & & \\
\hline T. ludeni (bean) - T. ludeni (cucumber) & -0.199 & 0.197 & 97 & -1.012 & 0.7426 & \\
\hline T. ludeni (bean) - T. urticae (bean) & -1.284 & 0.172 & 97 & -7.463 & $<0.0001$ & $* * *$ \\
\hline T. ludeni (bean) - T. urticae (cucumber) & -0.742 & 0.173 & 97 & -4.279 & 0.0003 & **** \\
\hline T. ludeni (cucumber) - T. urticae (bean) & -1.085 & 0.176 & 97 & -6.177 & $<0.0001$ & *** \\
\hline T. ludeni $(\mathrm{cu}$ & -0.543 & 0.1 & 97 & -3.068 & 0.0146 & * \\
\hline T. urticae (bean) - T. urticae (cucumber) & 0.542 & 0.149 & 97 & 3.629 & 0.0025 & $* *$ \\
\hline \multicolumn{7}{|c|}{ B. Signature of the ghost competitor on performance of $T$. urticae } \\
\hline T. urticae (comp.) - T. urticae (no comp.) & -0.034 & 0.063 & 325 & -0.544 & 0.8498 & \\
\hline T. urticae (com & 0.475 & 0.065 & 325 & 7.299 & $<0.0001$ & *** \\
\hline T. urticae (no comp.) - T. ludeni (no comp.) & 0.510 & 0.066 & 325 & 7.756 & $<0.0001$ & $* * *$ \\
\hline \multicolumn{7}{|c|}{ C. Performance of $T$. urticae } \\
\hline \multicolumn{7}{|c|}{ Investigate local adaptation (pooled across time points) } \\
\hline T. urticae (no comp.) - T. urticae (comp.) & 0.1119 & 0.0515 & 453 & 2.174 & 0.0767 & \\
\hline T. urticae (no comp.) - T. urticae (control) & 0.1605 & 0.0516 & 453 & 3.110 & 0.0056 & ** \\
\hline T. urticae (comp.) - T. urticae (control) & 0.0486 & 0.0516 & 453 & 0.941 & 0.6144 & \\
\hline
\end{tabular}

4 


\section{Table 4(on next page)}

Summary of the final best-fitting GLMM explaining reproductive performance

The values provided in the table are the raw and untransformed estimates due to the negative binomial distribution in the model. 
1 Table 4:

2 Summary of the final GLMM explaining reproductive performance. The values provided in

3 the table are the raw and untransformed estimates (negative binomial distribution).

\begin{tabular}{|c|c|c|c|c|c|}
\hline & Estimate & $\mathrm{SE}$ & $z$ value & $P$ value & \\
\hline \multicolumn{6}{|c|}{ The dynamics and performance of the ghost (fecundity at first measured time point) } \\
\hline (Intercept) (T. ludeni on bean) & 2.5867 & 0.1367 & 18.92 & $<2 \mathrm{e}-16$ & $* * *$ \\
\hline Cucumber & 0.1990 & 0.1965 & 1.01 & 0.3114 & \\
\hline T. urticae & 1.2838 & 0.1720 & 7.46 & $8.47 \mathrm{e}-14$ & $* * *$ \\
\hline Cucumber : T. urticae & -0.7407 & 0.2468 & -3.00 & 0.0027 & $* *$ \\
\hline \multicolumn{6}{|c|}{ Signature of the ghost competitor on performance of $T$. urticae } \\
\hline \multicolumn{6}{|c|}{ Fecundity assessed on bean (pooled across time points) } \\
\hline (Intercept) & 3.7098 & 0.0899 & 41.27 & $<2 \mathrm{e}-16$ & \\
\hline \multicolumn{6}{|c|}{ Fecundity assessed on cucumber (pooled across time points) } \\
\hline (Intercept) & 3.2209 & 0.0883 & 36.47 & $<2 \mathrm{e}-16$ & $* * *$ \\
\hline Initial density $T$. ludeni & 0.0693 & 0.0298 & 2.33 & 0.0199 & $*$ \\
\hline \multicolumn{6}{|l|}{ Density after plateau phase } \\
\hline (Intercept) (T. ludeni without comp.) & 2.5095 & 0.0539 & 46.55 & $<2 \mathrm{e}-16$ & $* * *$ \\
\hline T. urticae with comp. & 0.4751 & 0.0651 & 7.30 & $2.91 \mathrm{e}-13$ & $* * *$ \\
\hline T. urticae without comp. & 0.5095 & 0.0657 & 7.76 & $8.79 \mathrm{e}-15$ & $* * *$ \\
\hline \multicolumn{6}{|c|}{ Performance of $T$. urticae (fecundity pooled across time points) } \\
\hline (Intercept) T. urticae without comp. & 3.5241 & 0.0395 & 89.23 & $<2 \mathrm{e}-16$ & $* * *$ \\
\hline T. urticae under comp. & -0.1119 & 0.0515 & -2.17 & 0.0297 & $*$ \\
\hline T. urticae control & -0.1605 & 0.0516 & -3.11 & 0.0019 & $* *$ \\
\hline
\end{tabular}

4 


\section{Table 5 (on next page)}

Model selection (A) and Wald $\chi^{2}$ test (B) for the influence of total initial density on fecundity

Overview of the best models based on the lowest AICC with an AICC weight of at least 0.100. 
1 Table 5:

2 Model selection (A) and Wald $\chi^{2}$ test (B) for the influence of total initial density on fecundity.

3 Overview of the best models based on the lowest AICc with an AICc weight of at least 0.100.

\begin{tabular}{|c|c|c|c|c|c|}
\hline Model & df & LogLik & $\mathrm{AICc}$ & $\mathrm{AICc}$ & AICc weight \\
\hline \multicolumn{6}{|c|}{$\begin{array}{l}\text { Fecundity assessed on bean - max. model: fecundity } \sim \text { time }+ \text { total initial density }+ \text { time : } \\
\text { total initial density }+(1 \mid \text { block/island })\end{array}$} \\
\hline No fixed effects & 4 & -768.410 & 1545.1 & 0.00 & 0.293 \\
\hline Total initial density & 5 & -767.371 & 1545.1 & 0.04 & 0.287 \\
\hline Time & 8 & -764.312 & 1545.5 & 0.45 & 0.235 \\
\hline Time + total initial density & 9 & -763.522 & 1546.2 & 1.10 & 0.170 \\
\hline
\end{tabular}

Fecundity assessed on cucumber - max. model: fecundity $\sim$ time + total initial density + time : total initial density $+(1 \mid$ island $)$

$\begin{array}{llllll}\text { No fixed effects } & 3 & -597.445 & 1201.1 & 0.00 & 0.617\end{array}$

$\begin{array}{llllll}\text { Total initial density } & 4 & -597.132 & 1202.5 & 1.48 & 0.294\end{array}$

\begin{tabular}{llccc}
\hline B) & Independent variables & Chisq & Df & Pr(>Chisq) \\
\hline Fecundity on bean & Time & 8.3414 & 4 & 0.0798 \\
& Total initial density & 1.5149 & 1 & 0.2184 \\
& Time : total init. dens. & 4.4325 & 4 & 0.3506 \\
Fecundity on & Time & 4.2049 & 4 & 0.3790 \\
cucumber & Total initial density & 0.7819 & 1 & 0.3766 \\
& Time : total init. dens. & 3.8180 & 4 & 0.4312 \\
\hline
\end{tabular}

4 